\title{
BOOM IN DEMAND FOR GENETICS SERVICES IN NSW
}

Jennifer Blackwell, Executive Officer, NSW Genetics Service, NSW Health Department

G enetic disorders and birth defects impose a heavy physical, emotional and financial burden on individuals, families and the community. Advances in science and technology are expanding knowledge of the role of genes in disease and are leading to better methods of preventing, diagnosing and treating genetic disorders and diseases of multiple causes, including genetic factors. Developments in screening programs, prenatal diagnosis and community education are increasing the availability of, and demand for, genetics services.

A major challenge is to implement new genetic technology in the most beneficial way, to ensure high-quality care and to ensure access to services. A new five-year plan, 1993 1998 , provides a framework for the ethical and effective development of genetics services until 1998. Maintaining the principle of an integrated approach encompassing clinical, counselling, educational and laboratory services centrally coordinated through the NSW Genetics Service Advisory Committee - is seen as the most effective and economical way of achieving the goals of the NSW Genetics Service. The major goals include:

口 ensuring the provision of, and access to, genetics services appropriate to the needs of the people of NSW; improving the use of information and services
relating to genetic disorders and birth defects; and reducing the impact of genetic disorders and birth defects on the sufferers, individuals at risk and their families.

Genes contain the information that determines how we grow, what we look like and how well our bodies work. Information in a gene is called the genetic code. Genes are part of chromosomes which are in all the cells of our bodies. We each have 46 chromosomes which contain between 50,000 and 100,000 genes. Chromosomes and genes are made of DNA. Our body's health and growth will be changed if the genetic code is changed or if there are extra or missing genes.

There has been an explosion of knowledge about the processes of heredity and their implications for human health. The international Human Genome Project aims to identify all the 50,000 to 100,000 genes in humans by the year 2005 .

Using both molecular biology techniques and family studies, nearly 5,000 disorders have been identified as being caused by a mutation in a single gene. The number of disorders susceptible to molecular genetic or DNA diagnosis is rapidly increasing. In recent months, genes have been located for Huntington disease, motor neurone disease, melanoma and osteoporosis.

Many medical problems evident at birth, or soon after birth, are primarily genetic in origin or include a genetic factor. Other genetic disorders appear later in life. Genetic disorders include: Down's syndrome, neural tube defect, cystic fibrosis, diabetes, fragile $\mathrm{X}$ syndrome, muscular dystrophies, spinal muscular atrophies, haemophilia, hearing disorders, neurofibromatosis, polycystic kidney disease, thalassaemias, phenylketonuria, galactosaemia and Huntington disease.

They also include some forms of heart disease, cancer, asthma, epilepsy, visual disorders, short stature syndromes, intellectual disability and hypothyroidism.

\section{Aboriginal mothers and babies}

\section{$\checkmark$ Continued from page 3}

The risk of perinatal death is higher for infants born prematurely. Seventeen per cent of Aboriginal infants born prematurely died in the perinatal period. This was 1.4 times higher than for non-Aboriginal premature births. However, excluding infants born at less than 25 weeks gestation, Aboriginal preterm births were no more at risk of perinatal death than non-Aboriginal preterm births. This suggests that at least some of the excess risk of perinatal death for Aboriginal infants is due to the fact that Aboriginal infants are more likely to be born prematurely.

The risk of perinatal death for Aboriginal births was highest for teenage mothers (30.3 per 1,000 births) (Figure 4), women having their first child ( 34.0 per 1,000 births), and vaginal breech deliveries (310.3 per 1,000 births).

Extreme prematurity was the single most common cause of perinatal death for Aboriginal infants, accounting for 19 per cent of perinatal deaths. In contrast, extreme prematurity accounted for 12 per cent of non-Aboriginal perinatal deaths. The rate of perinatal death due to extreme prematurity for Aboriginal births was 4.5 per 1,000 births, which was 3.0 times higher than for non-Aboriginal births.

'Maternal conditions which may be unrelated to pregnancy' were associated with 17 per cent of Aboriginal and 10 per cent of non-Aboriginal perinatal deaths. Most of these maternal conditions were hypertensive disorders (high

\section{FIGURE 4}

PERINATAL MORTALTTY BY ABORIGINALITY AND MATERNAL AGE, NSW 1987-90

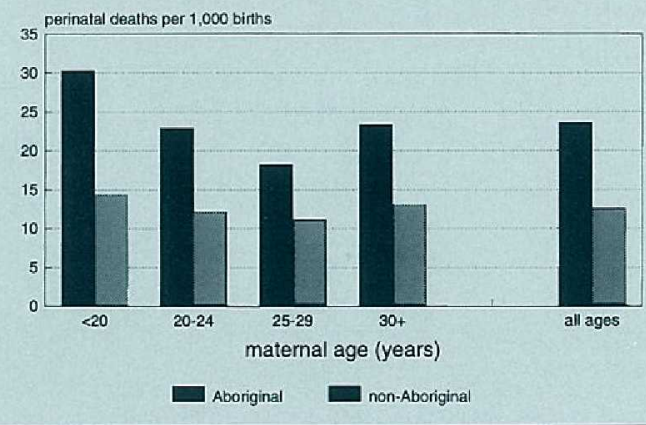

blood pressure). The perinatal death rate associated with maternal hypertensive disorders for Aboriginal births was 2.2 per 1,000 births, which was 3.1 times higher than for non-Aboriginal births.

'Other placental separation and haemorrhage' (i.e. other than placenta praevia) and 'other and unspecified morphological and functional abnormalities of the placenta' were both associated with 12 per cent of Aboriginal perinatal deaths. The perinatal death rates associated with these conditions for Aboriginal births were 2.1 and 3.6 times higher, respectively, than for non-Aboriginal births. 
At least one in 20 people will experience a gene-related disability by the age of 25 . In the average lifetime, more than half the population will have a disease with a genetic component. Many chronic diseases of middle and old age appear to be due to multiple causes, including genetic factors. Up to half the admissions to paediatric hospitals, and 12 per cent of adult admissions to general hospitals, are for genetic disorders and about 28 per cent of infant deaths are due to birth defects.

Like newborn screening for phenylketonuria, cystic fibrosis, galactosaemia and hypothyroidism, early detection means effective treatments can be introduced both to relieve suffering and reduce the high costs associated with the long-term care of individuals with chronic disability.

Information and counselling can enable couples at genetic risk to have healthy children through informed choices about reproductive options, including prenatal diagnosis. Many congenital malformations, chromosomal abnormalities and metabolic errors can be diagnosed early in pregnancy. Prenatal testing is widely available for two of the most common disorders, Down's syndrome (among women of advanced maternal age) and neural tube defect.

Prenatal molecular genetic diagnosis is available for couples where the pregnancies are at high risk for disorders such as cystic fibrosis, Duchenne/Becker muscular dystrophy, myotonic muscular dystrophy, polycystic kidney disease, spinal muscular atrophy, Huntington disease, haemophilia, thalassaemia and fragile $\mathrm{X}$ syndrome. Long-term social and financial benefits can be achieved through prenatal diagnosis. Carrier testing and presymptomatic diagnosis are also available.

Screening for genetic predisposition could achieve early intervention or prevention of some disorders, reducing distress and costs associated with later complications. There is a genetic component in the cause of many common conditions such as coronary artery disease, diabetes and some cancers, including melanoma, breast and bowel cancer. Dietary or lifestyle changes can be effective. For example, those identified at high risk of lung cancer could reduce the likelihood of developing the disease by avoidance of cigarette smoking. Identification of those at increased cardiovascular risk means dietary and lifestyle modifications can be adopted to reduce risk. Where parents are affected, early identification and intervention will benefit their offspring.

Public education is vital so people are aware of the options available to them to reduce disability.

Pregnancy often provides the first occasion when couples consider reproductive risks related to family history or age, so awareness by general practitioners and obstetricians is especially important. Clients at risk for a genetic disorder or at risk of having a child with a birth defect or genetic disorder can be referred to specialist genetics services for evaluation and counselling.

Clinical services operate principally through four major clinical genetics units: Prince of Wales Children's Hospital (POWCH), Royal Alexandra Hospital for Children (RAHC), Westmead Hospital and in the Hunter Area. These units provide a Statewide network of services to metropolitan and country centres and at prenatal diagnosis clinics in level 5/6 maternity hospitals. In NSW there are nine clinical geneticist positions and four training posts.

The value of non-medical genetic counsellors in making services more accessible has been recognised with the employment of 22 ( 15 full-time equivalent) counsellors, in the major units and at the Royal Hospital for Women, Royal
North Shore Hospital, Wollongong, Gosford, Lismore, Coffs Harbour, Port Macquarie, Tamworth, Broken Hill, Dubbo, Goulburn and Wagga. Genetics clinics are held at King George V, Liverpool and Nepean hospitals. Clinical geneticists and genetic counsellors also hold clinics in other country towns.

A limited number of laboratories provides a Statewide service. Cytogenetics laboratories are associated with each clinical genetics unit at POWCH, RAHC, Westmead and in the Hunter Area. Molecular genetics services operate on a one disorder/one laboratory consortium model where each disorder is studied in only one laboratory in NSW. Statewide services are provided from three laboratory groups: POWCH/Concord, RPAH/RNSH and Hunter. Biochemical genetics and the newborn screening program are at the Oliver Latham Laboratory.

The Statewide genetic education program is based at Royal North Shore Hospital.

The NSW Genetics Service Advisory Committee, in line with international guidelines, recommends a ratio of one geneticist per 300,000 population. To achieve this standard, a further 14 geneticists will need to be trained and employed over the next five years. Based on demographic and geographic factors in NSW, it is recommended that by 1997 three additional clinical genetics units be established in the Northern Sydney Area, Liverpool and Royal Prince Alfred/King George V (RPAH/KGV) hospitals, making a total of six units (assuming Westmead and RAHC become one unit from 1996). A clinical geneticist position is recommended for Wentworth Area with joint appointment at a major unit.

The employment of additional genetic counsellors throughout the State will improve access to information and make services more effective and more economical. Development of services is desirable in Wentworth, South Western Sydney, Southern Sydney, Central Sydney and in Western Sydney.

Expansion of cytogenetics laboratory staff will be required to handle increasing demand for prenatal studies, and particularly at RNSH to support the new clinical genetics unit. Clinical genetics units at Liverpool and RPAH will use existing services for the term of this five-year plan.

Another molecular genetics collaborative group is planned for RAHC/Westmead/Liverpool by 1996-97. The number of hospital scientists will need to rise to manage demand for DNA testing.

Newborn screening is a single Statewide service and would be most appropriately attached to a major children's hospital. This will be achieved with the relocation of Oliver Latham Laboratory to RAHC, with the biochemical genetics services provided by that laboratory.

The Statewide genetic education program will continue to promote community awareness of genetic health issues and of preventive services. The program will develop resources for incorporation into school curricula and community education services. The program also is responsible for keeping health professionals informed of developments and new services.

The increasing importance of DNA technology, diagnosis and potential treatment should encourage Health Areas and Districts to enhance services for their preventive, therapeutic and counselling values. Investment in genetics services is offset in the long term by the reduction in the incidence of chronic conditions and that cumulative savings will be in the interests of health services and the communities they serve. 